\title{
ROUTE CHOICE MODELLING FOR BICYCLE TRIPS
}

\author{
Ehsan Beheshtitabar ${ }^{1}$, Sergi Aguilar Ríos ${ }^{2}$, David König-Hollerwöger ${ }^{3}$, Zdeněk Svatý4, \\ Clas Rydergren ${ }^{5}$ \\ 1,2, 3, 4,5 Department of Science and Technology (ITN), Linköping University, 58183 Linköping, Sweden
}

Received 14 March 2014; accepted 23 April 2014

\begin{abstract}
This paper intends to find out which parameters have the highest influence on bicyclists' route-choice-behaviour and how they contribute. There are many attributes considered throughout different studies that are favoured by cyclists. The attributes are the basis for generating a function which predicts the route choice of cyclists. The paper aims at making a comparison between distance-based routes and attribute-based routes. The model for creating the bicycle route choice program is based on the network model of Norrköping, Sweden. The preferred attributes of the model each assign a weight in the cost function of the model for each link of the urban network. The algorithm of the lowest cost function route searches the shortest path in terms of assigned link costs over the whole network. For comparing the results of the cost function and the shortest route (between an origin-destination), the model has a shortest path finding algorithm between different Origin and Destination pairs implemented. It has been viewed that around $25 \%$ of all cumulative routes do not show any distance differences between the shortest path distance and attributes based solution. With the used weights of the Standard scenario, it can be seen that the trade-off between shortest distance and safety respectively comfort can be optimized, fulfilling both criteria (distance and safety/comfort).
\end{abstract}

Keywords: bicycle route choice, attributes, cost function, modeling.

\section{Introduction}

In the modern day traffic administrations, cycling has gained higher importance than the past. Due to numerous social, environmental and economic issues raised, cycling is promoted more and more by traffic planning authorities. This is caused by obvious advantages of cycling: healthy, quiet, cheap and not polluting mode of transportation. Therefore it is quite reasonable that in future there will be a greater share of bicyclists observable. Nowadays there is not an adequate tool existent which could be used to predict bicyclist route choice, taking into account all important attributes to model bicyclists' behaviour as real as it gets. Existing models focus on few main attribute for route choice such as distance, or travel time. Though these models seem reasonable, they still have the capacity for improvement and progress.

\subsection{Aim and Purpose}

The aim of the paper is to find out which parameters have higher influence on bicycle route choice, and how they are contributing. For this purpose, a link-based cost function, field tests as well as computer simulations are defined with the help of the literature review. Such a cost-function is the base for further modelling activities. So it is conceivable to embed such a function into

\footnotetext{
${ }^{1}$ Corresponding author: ehsbe019@student.liu.se
} 
already existing transport models. As a result policy makers could trust on more detailed data. Further also traveler information systems would be able to apply such a modelbased framework.

The result of the paper is, on the one hand, a model capable to predict the route choice for commuters. This could be helpful, for example, in order to plan new infrastructure investments or traffic planning. On the other hand, a useful tool for trip planning is also presented. The resulting product of this paper is a program with a friendly user interface. A bicyclist without any previous knowledge of the network can choose any origin and destination and will obtain the shortest route between these two points, and the most suitable one according to the presented cost function.

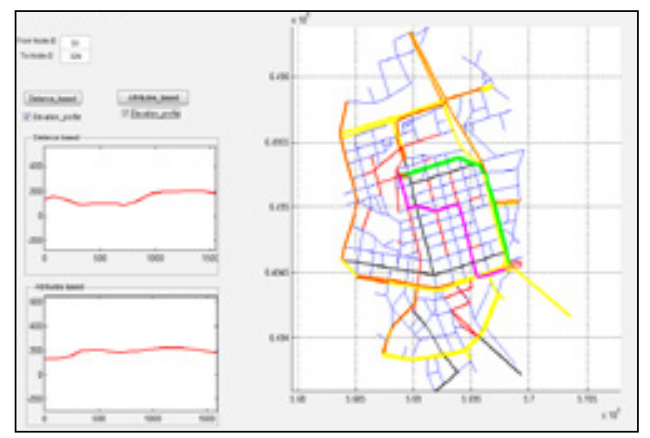

Fig. 1.

GUI Bicycle-Route-Choice Program

Fig. 1 shows exemplary a clipping of the paper model output. Hereby on the left part of Fig. 1 the elevation profiles of two different route choices are shown. On the right side these two according paths - green (upper elevation profile) and magenta - are displayed.

The contribution of this paper to the topic is basically a new cost function capable to predict the bicycle route choice of a commuter, based on the attributes that were considered most important. Also presented is a detailed network for Norrköping downtown, which further usage is discussed in the end of the report.

\section{Background}

In the beginning previous sources and literature which have dealt with the cyclist route choice prediction, usually based on some already observed and realized routes are pointed out. In the next sections a survey of existing cycle planners which has been carried out within this paper is discussed. Under the term of the cyclist prediction it is meant recognizing tools able of recognition of route choices for generalized and universal cyclists which are in same target group, such as commuters. The term cycle-planners covers mostly searching tools able to find ideal route based on user preferences which are necessary to be input before carrying out the results.

\subsection{Cyclist Route Choice Prediction}

In the literature, some bicycle routing models being under research were reviewed. In these articles, the authors have investigated lots 
of various attributes, which for the means of cyclist route choice are relevant or important. Since some research studies are a case study or are dealing with a specific environment of cycling, they have certain perceptions for attributes, but mostly the main attributes considered are length or travel time, gradient, existence of bicycle facility such as cycle lanes, intersections, age and experience of cyclist and traffic volume. Sometimes this kind of modelling has been studied altogether with pedestrian traffic (Westerdijk, 1990). Goldsmith (1992) depicts cyclist routings as, "The consideration of factors beyond travel time and distance are particularly important for bicycling as cycling levels are considered to be also affected by many additional characteristics of the network such as road type and automobile volumes". This gives a clear vision that unlike vehicle routing, cyclists don't have the time-distance criteria as the main priority, and a few quality matters, concerns their route-choice. In some cases, cyclist's shortest route to destination would have to pass over a rail track or other obstacles, which would be avoided by cyclists.

Other articles assume that most bicyclists take the shorter distance path or that they tend to avoid grade differences. As examples of literature which is dealing with attributes in different studies, Sener et al. (2008) or Hunt and Abraham (2007) can be mentioned.

As a matter of fact, many attributes can be considered, although there are some of them which are present in almost every study because of their relevance. Those are the ones chosen for this project, but this will be further discussed in the section dedicated to attributes. Many articles try to figure out the best way of represent which is the main factor affecting bicycle route choice. For instance, Ehrgotta et al. (2012) develop a cost function based on an attribute called "suitability". This qualitative measure is compounded by twenty road factors such as motor traffic volume and speed, slope, percentage of heavy vehicles, lane width or cycle facilities.

One of the major points of research and modelling is data collection. This has been done in many different approaches, including surveys, real-time observations, etc. Sener et al. (2008) have drawn cyclist's data from a web-based stated preference survey of Texas bicyclists.

Stinson and Bhat (2003) have developed their bicycle route choice for only commuters. They have created empirical models based on Stated Preference (SP) survey over the internet. SP surveys are surveys where respondents are presented with several hypothetical route choices. In the survey they have defined eleven determinants for route-choice decisions and included a comprehensive set of linklevel and route-level factors. For obtaining detailed and reliable data they defined nine different survey instruments, where each instrument had few attributes considered mainly, and a few scenarios inside the instrument being the effect of varying attributes of that instrument. The eleven attributes where hidden in the scenarios of the instruments. The authors have divided the attributes to link-level factors, as the attributes defining the link characteristics, (Roadway class, Parallel parking permitted, Bicycle facility type, Bridge type, Hilliness, Riding surface) and route-level factors for attributes affecting the overall route choice (Travel time, Facility continuity (yes/no), Number of stop signs per mile, Number of 
red lights, Number of major cross-streets). After the survey, their data had been used for discrete-choice modelling. For their model, they have defined a binary logit model, which inputs the data of survey for each individual and has an output which is the utility of that route for that certain individual. Their results indicate that travel time is the most important factor for cycling commuters and after that the presence of any type of bicycle lane is decisive for commuter route-choice. On the other hand, the delays caused by traffic signals, presence of parking facilities alongside a route and the range of gradient change through the route where the major factors that commuters evaluated about their route.

Broach and Gliebe (2007) has collected survey data from 162 cyclists with GPS tracking devices. These research articles show the diversity of methods in data collection and the different approaches towards processing these data for a cycler's routing model.

\subsection{Survey of Current Models}

Within literature research was carried a survey of already existing planners for cyclist. In upcoming part are given examples of currently existing route planners. Main intention was not to cover all existing ones but present different approaches and ways of route planning. Several cycle planners, mostly for cities, already exist and are available online nowadays. Planners were created, mostly within bigger programs which tried to promote cycling, healthy way of living or at least as improvement of city tourist services, and developed mostly by universities which were cooperating with municipalities or government.
The first group of cycle planners are ones which are covering travelling around the city. An example which could be used is the Vancouver cycle planner (Cycling Route Planner, 2007). The user has a possibility to set his preferences, such as priority usage of cyclist facilities instead of major roads, shortest path, least elevation gain path, least traffic pollution or mostly vegetated path. Then the planner finds ideal path which is based on these preferences and display the path on the map with overall information about it along with directions. Another even more detail approach is used in the OPT for health - route planner for San Francisco (OPT for Health, 2010). Cyclists are not the only target group but possibility to adjust the searching for ideal route is here even more complex. The user is allowed to set values for each attribute which is considered (e.g. most bicycle friendly, major road, traffic access restricted...). This setting is supposed to lead to improvement of searched path. On the other hand there are other types of cycle planners which are trying to be still complex even with limited amount of adjustable preferences such as San Francisco Bicycle trip planner (2009). In this planner the user can choose only from three types of paths - shortest, balanced or biker friendly and setting of maximum grade.

Currently there are also some planners which are trying to cover larger areas than cities. One of those is AnachB (2006) which claims to be permanently updated planner for all means of transport in Vienna, Lower Austria and Burgenland. The cyclist can select here again from three possible alternatives - Leisure (preference for cycle infrastructure, special preference for cycle paths, cycle lanes and routes without motorized traffic, avoid steep climbs, cycling speed $15 \mathrm{~km} / \mathrm{h}$ ), Advanced (preference for 
cycle infrastructure, avoid steep climbs, cycling speed $20 \mathrm{~km} / \mathrm{h}$ ) or 'My trip' where can cyclist adjust some of the criteria and set the cycling speed (preference for cycle infrastructure, OR/AND special preference for cycle paths, cycle lanes and routes without motorized traffic OR/AND avoid steep climbs). Afterwards basic information about trip (travel time, distance) is displayed and the map can be downloaded in pdf format. Another example of large area covering route planner can be Transport direct (2003) which is covering route planning around Britain. Selectable criteria in default are Quietest or The Most recreational but with possibility of further possibility to avoid unlit roads, walking bike or time based restrictions. Only limitation is that cyclist trip cannot be longer than 50 miles. After ideal route is found the planner displays map, basic information and elevation profile of the route.

To sum up it is possible to note that most of currently existing and working route planners for cyclist incorporate possibility to set preferences of the cyclist. Some of them are too detail in demand of the input information and some of them are on the other hand to general. Furthermore almost none of these cycle planners have their functions and principles shown or accessible by public or any researchers. Therefore is really hard to evaluate, compare or use them in development of really feasible and reliable cost function. But some trends are visible, basic attributes and important issues which are commonly used.

\section{Model Creation}

The model for creating the bicycle route choice program is based on the network model of Norrköping. The model itself has a shortest path finding algorithm between different Origin and Destination pairs implemented. This algorithm looks for the shortest path in terms of assigned link costs over the whole network. Therefore a cost-function for defining the total links costs was defined as described later. To evaluate afterwards all different Origin and Destination combinations the concept of Floyd and Warshall for finding the shortest respectively least costs path was applied, since this algorithm already stores all different route combinations and the resulting costs. Further this concept provides routing, based on directed and undirected graphs. The only precondition for this algorithm is, having positive assigned linkcosts, which was considered in the definition of the cost function.

To compare the results between pure distance based and attributes based routing there were accordingly two user options in the graphical-user-interface defined for visualizing the results.

\subsection{Network Design for Least Cost Function}

For simulating the route-choice behaviour of bicyclists there was chosen a networkbased-model approach. Therefore the geometrical as well as infrastructural information of the central area of the road network of Norrköping was extracted from a PostGis-server and was further utilized for the bicycling route-choice model.

Generally the decision for selecting the central area of Norrköping was based on the higher number of infrastructural attractions for bicyclists, including higher number of bicycle paths in the central region, higher assumed demand factors like schools, 
university, working and shopping facilities. Furthermore also different elevation profiles were occasional a reason for extracting this area of Norrköping considering the found important parameters (attributes) for the bicycle-route-choice model as described in the section of the attributes.
The map itself had to be transformed into a UTM (Universal Transverse Mercator)coordinate system to get distance-truth geometric data for evaluating shortest path based on link distances as well.

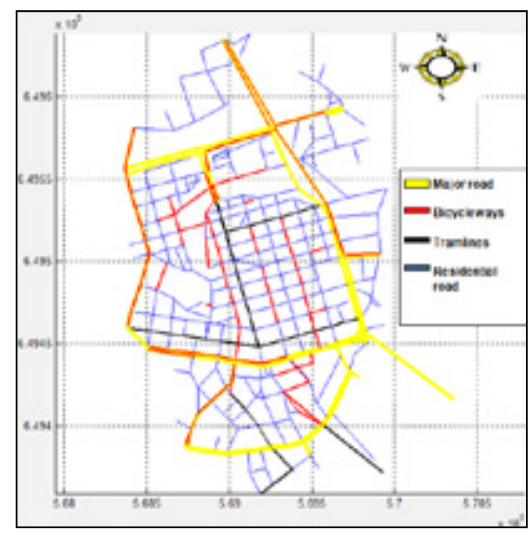

Fig. 2.

Map of Investigation Area of Central Norrköping for Bicycle-Route-Choice Analysis

Fig. 2 shows the overall used network for the bicycle route choice program. Basically 347 nodes respectively intersections were taken into account.

There are three different road types besides residential streets defined, these are: major roads, bicycle-ways and tramlines. In the later described cost function, major roads as well as bicycle paths play a decisive role in weighting the costs for each link. For the sake of simplicity all links were two-ways configured and the network itself is set up in a consistent way, which means that all nodes can be reached from one arbitrary node with different assigned paths. Under these circumstances the number of possible used O/D-pairs can be calculated with $\left(\mathrm{N}^{2}\right.$ $N)$, if $N$ is the total number of nodes.

\subsection{Data Collection and Availability}

The network data could be extracted from an universities' Postgis-database. Since the cost function also considers the steepness of bicycle routes, height information for each node was added additionally.

Therefore height data was extracted and assigned to each node from the Googleearth server. The information about road type could be used from OpenStreetMap (openstreetmap.org/) as well as Google-map web applications.

The following Table 1 represents the input data for the network, its entities and the resource. 


\section{Table 1}

Network Based Elements

\begin{tabular}{|l|l|l|}
\hline Element & Entity & Resource \\
\hline Road Geometry & UTM-zone $(33,66)$ & Postgis-server-University \\
\hline Bicycle Ways & $1 \ldots$ bicycle way, 2... separate bicycle-path & OpenStreetMap \\
\hline Height Data & Meters & Google Earth \\
\hline Major Roads & $3 \ldots$ if existing otherwise 1 & Google-Maps \\
\hline Trams & $2 \ldots$ if existing & OpenStreetMap \\
\hline Residential Roads & $1 \ldots$ if existing & Postgis-server-University \\
\hline
\end{tabular}

Since the provided information was partly inconsistent, some missing links had to be updated for providing a quite realistic and consistent network database for finding the cost-based paths. Generally there is one road type for each link assigned.

\subsection{Program Workflow and GUI Interface}

The program offers the user the possibility of selecting specific Origin and Destination nodes (O/D). Further it is the users' decision, whether a distance or an attributes costs based search should be conducted. As a result the shortest path depending on the selection is displayed. Because of comparison reasons both paths and its elevation profiles can be selected as well. The elevation profile is expressed in GUI by chart where the $\mathrm{Y}$-axis displays the elevation, and the $\mathrm{X}$-axis displays the distance. The following flow-chart diagram (Fig. 3) represents the program workflow and the database input.

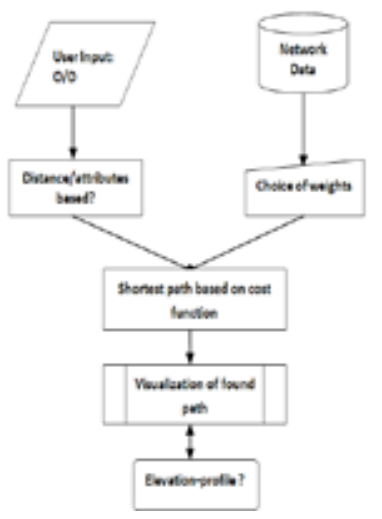

Fig. 3.

Program Workflow of Bicycle-Route-Choice Model

So the user has the opportunity to decide the starting and ending node (O/D) and further which costs based routing should be conducted as seen on the upper left part of the workflow diagram.

\section{Attributes}

Attributes can be defined as the factors which can be under consideration for preparing a model for cycling routing. Numerous 
attributes are potentially effective in the route-choice of cyclists, though some are of greater importance. Attributes can depend on many aspects, including a region, aim of cycling, age of cyclist, etc. As an example in the icy conditions of Sweden's winters, the safety of a route, taking into account, the least junction with other modes, or the least passing on ice, is a major priority for cyclists. Regarding these facts, there is the conclusion that, a certain network would favour certain attributes generally, with these attributes being the key ones, and the attributes which are preferred by a certain group are the more specific attributes. There is a limitation when dealing with attributes, and that is the process of integrating these attributes into a model. Apart from that, there is the issue of data availability, which for some attributes can never be precise, or logical, to implement into a numerical model. This would indicate that from all attributes potentially available, only a few will be integrated into the model. Regarding the facts mentioned above, there is some diversity in the attributes which have been considered in available models for routing cyclists. The attributes presented in present literature vary but are mostly:

- Distance,

- Gradient,

- Safety of route,

- Type of route

- Availability of separate cycle lane

- Junctions with public transport

- Number of junctions with traffic lights,

- Attractiveness,

- Quality of the road surface,

- Bicycle parking facilities.

In this paper, modelling a part of Norrköping city has been executed. The initial attributes which were thought to be significant were in their order, distance, slopes (for each link) and existence of cycle facilities.

The most crucial factor for route choice is obviously the distance. It is indirectly indicating travel time, but this can be altered by other attributes. Therefore, the shortest path is not always the fastest or the most "suitable". The slope is clearly an important attribute for route choice, since many cyclists think in taking a longer route in order to avoid steep hills. The last attribute included in this paper is the presence of cycle lanes within the path. Cyclists assume cycle lanes guarantee their safety more than sharing the route with other modes of transport.

The attributes considered for this paper, were supposed to be independent and each covering a major decisive factor in cycler's route. Since all the presented attributes have an impedance and also attractiveness, therefore they will have different rates of significance in the routing of cyclists. This is what is going to be monitored in the cost function proceeding in the next part of the paper. The weight considered for each attribute will be also discussed there. The other attributes observed were not appealed in the cost function, due to some limitations such as data availability and time.

\section{Definition of the Cost Function}

For creation of cost function, which is capable of predicting possible route choice of the cyclists, not only the travel time of possible routes should be taken into account (which is commonly used criteria for travel route choice), but also preference and decision affecting attributes which every cyclist take into account. In the literature there is often used the term 'suitability' (Ehrgotta et al., 2012) that is a qualitative measure for the 
safety and comfort for bicyclists contributing to their route-choice behaviour. To model the route-choice behaviour of bicyclists it is necessary to quantify this measure.

\subsection{Cost Function Formula}

With the help of literature review, already used bicycle-route-choice models and the aspects of safety and comfort for bicyclists it is decided to use the Eq. (1) cost function model which takes into consideration not only travel time, expressed by length of the route, but also other attributes which are unified under the term 'suitability'.

Path index $=\min _{k} \sum_{i \in P_{k}} d_{i}\left(\alpha+\beta \cdot s l_{i}+\gamma \cdot c f_{i}+\delta \cdot r t_{i}\right)$

Where $\mathrm{k}$ are the indexes of all founded paths $\mathrm{P}$ that are consisting from links with indices $i_{1}, i_{2} \ldots i_{N}$ and are connecting selected $\mathrm{O} / \mathrm{D}$ pair, $d_{i}$ is length of segments $i, s l_{i}$ is grade observed on segment $i, c f_{i}$ is value describing existence of cyclist facilities, $r t_{i}$ is road type present on segment $i$ and $\alpha, \beta, \gamma$ and $\delta$ are weights for each attribute.

The reason why distance was taken into account instead of travel time is that there is the assumption that cyclists travel with fixed speed across the whole network. In this paper a cyclist's travel time is based purely on distance. In reality are cyclist affected by elevation profile but it was decided to use the effect of slope separately in other part of function. Furthermore it can be mentioned that cyclist are not influenced by traffic jams or do not have to adjust their speed to traffic signs and therefore is their speed much more consistent than in other modes of transport. The term of 'suitability' is represented in this function by three main attributes which are having weight on the cost function: slope, presence of cyclist facility and route type on each link.
For each of these parameters, a different weight has been considered in pre-evaluation state. After that it was evaluated with many different weights for each attribute in order to find out the ideal cost function for bicycle route choice. All weights have same effect on each link in the model independently on length of the segment. This is achieved by multiplication by distance within the formula.

\subsection{Used Attributes}

Length $\left(d_{i}\right)$ of the link is one of the most important attributes even though not the only one. It can be noted that most of the road users in the target group think about taking the shortest path before starting the trip and then adjust the route based on already mentioned 'suitability'. Influence of length and tendency of preferring of the shortest path is in the cost function represented by weight $a$. As was mentioned before distance of the segment is used in formula as well for equal assignment of the costs for each link. Based on the literature studies it was found that people prefer to use alternative routes which can be longer than shortest path but until certain point. This fact was later implemented and considered within the evaluations.

First parameter covering the term of 'suitability' was slope. Again based on literature survey it was found that how grade of slope is affecting cyclists, their speed and style of driving (Hills, Gradients \& Slopes, 2012; Roberts, 2012). Then several groups of grade which have different effect on the cost function were defined. These groups are represented as intervals within predefined bordering values. Used bordering values of grade were $-10 \%,-2 \%, 0 \%, 2 \%, 6 \%$ and $10 \% .1 \%$ of the grade is adequate to elevation 
gain 1 meter on 100 long road stretch. The effect of the slope is then represented in the cost function by actual value of grade in percent together with weight $\beta$. The value of the weight differs for each group and therefore ensures if the slope will have positive or negative effect on resulting link cost. Overall within the cost function are preferred routes with minor elevation gain and is favoured downhill slope. Furthermore high values of grade are highly penalised within the function.

By the term cyclist facility $\left(c f_{i}\right)$ the existence of any cyclist lane without distinguishing is meant, whether it is separate lane next to sidewalk, marked space for cyclist on the road or any other type. This limitation was caused by data set, which was obtained from the OSM database. In the cost function it was decided to favour existence of any type of cyclist facility and this favour is then represented in function by weight $\gamma$. This is based on literature studies and surveys such as Westerdijk (1990) or Menghini et al. (2010), where was shown that cyclist prefer to use cyclist facilities.

Last attribute that was considered as important and is implemented into the cost function is type of the road $\left(r t_{i}\right)$ existing on link. Based on data set which was obtained two main road types were distinguished - residential and secondary roads - with additional information about existence of tram traffic. Reason is that existence of tram traffic or higher volumes of traffic occurring on secondary roads are affecting perceived safety of the cyclist, especially when they are forced to share space with this type of traffic. In function is therefore road type considered only in case that there is secondary road or tram traffic on the segment observed without any cyclist facility and is then penalized within the function. Penalization is expressed by weight $\delta$.

\subsection{Possible Future Improvements}

Only the attributes mentioned above were included within the cost function because of several limitations. First one was caused by insufficient data which were obtainable. Data set was not detail enough to implement further attributes such as winter maintenance, more detail road type or cyclist facilities division with possible different weighting. Another limitation was caused by much more complex and demanding programming which would allow more detail alternative route creation and evaluation. Nevertheless it can be said that the function in current form is ready for any another further implementation of another attributes by adding them together with already implemented ones.

\section{Evaluation Results}

Basically the evaluation of the bicycle-routechoice model is based on the test subject's evaluation and calculation results. Besides testing elasticities, Standard scenario, with the weighting factors as described in the cost function section, was applied. Further differences in calculated distance and attributes paths are analysed. Therefore a cumulative distribution function (CDF) was applied and used for evaluating all possible routes combinations.

\subsection{Weight Values Validation and Evaluation}

As was already mentioned for the first set of tests where used pre-set values for each attribute based on presumed importance to the route choice and literature research. 
Afterwards each member of the group separately experimented with numerous different $\mathrm{O} / \mathrm{D}$ pairs and compared obtained results with his experiences, preferences and expected results. Within the program followed factors were mainly preference and usage of the cycle facilities, the elevation gain and the reasonability of used links. Tested $\mathrm{O} / \mathrm{D}$ pairs were selected with intention of covering of all possible variations within tested network.

An example of $\mathrm{O} / \mathrm{D}$ pair is presented below. In Fig. 4, routing results of attribute based and shortest path computation between nodes 91 and 329 together with the elevation profiles of each path are displayed. In the example it can be seen that the attribute based path, marked with magenta colour, is showing an increasing utilization of bicycleway equipped links and have improved elevation gain and profile throughout the whole route. Afterwards Table 2 presents overall length of both paths together and distances between each nodes within founded paths. Resulting values of path length are showing that attribute based path is longer only by 43.1 meters which is $3 \%$ increase of length in comparison to shortest founded path.

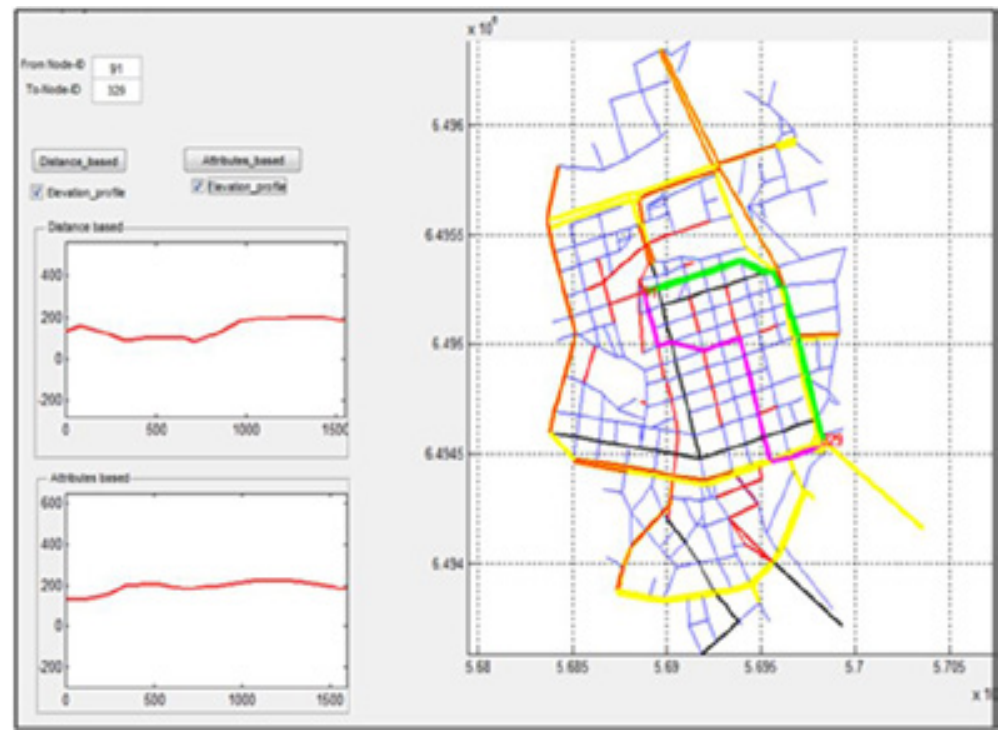

Fig. 4.

Example of Founded Paths between Nodes 91 and 329 
Table 2

Path Results

\begin{tabular}{|c|c|c|c|}
\hline \multicolumn{3}{|c|}{ Path results - FROM node ID: 91, TO node ID: 329 } & Distance [m] \\
\hline Node ID - distance based & Distance [m] & Node ID - attributes based & 0 \\
\hline 91 & 0 & 91 & 85,6 \\
\hline 115 & 77,6 & 97 & 85,5 \\
\hline 165 & 153,9 & 108 & 83,6 \\
\hline 190 & 97,5 & 113 & 76,6 \\
\hline 217 & 99,9 & 143 & 174,3 \\
\hline 237 & 105,4 & 188 & 97,1 \\
\hline 268 & 133,5 & 213 & 101,6 \\
\hline 279 & 44,5 & 235 & 78,5 \\
\hline 288 & 87,3 & 239 & 88,4 \\
\hline 292 & 13,6 & 246 & 87,1 \\
\hline 302 & 153,9 & 252 & 103,1 \\
\hline 306 & 92,2 & 257 & 109,9 \\
\hline 307 & 13,9 & 263 & 118,0 \\
\hline 315 & 162,2 & 272 & 107,1 \\
\hline 325 & 217,7 & 298 & 125,0 \\
\hline 329 & 95,4 & 319 & 46,5 \\
\hline
\end{tabular}

Based on these observations and evaluations which were described above were adjusted weights for each attribute.

Table 3

Final Evaluated Weight Values Used

\begin{tabular}{|c|c|c|}
\hline Weight & Measured value of attribute & Weight value \\
\hline $\mathrm{A}$ & & 0,7 \\
\hline & above $10 \%$ & 0,03 \\
& $(10 \%, 2 \%)$ & 0,02 \\
$\mathrm{~B}$ & $(2 \%, 0 \%)$ & 0,01 \\
& $(0 \%,-6 \%)$ & 0,03 \\
& $(-6 \%,-10 \%)$ & $-0,01$ \\
& below $-10 \%$ & $-0,03$ \\
\hline$\Gamma$ & & $-0,25$ \\
\hline$\Delta$ & & 0,1 \\
\hline
\end{tabular}

\subsection{Elasticity of Different Weights - Scenario Testing}

For testing the elasticity's of the cost function, four different scenarios were defined. The relation between the number of changed routes attributes based - if all $\mathrm{O} / \mathrm{D}$-pairs are computed - to the total number of all purely shortest-distance based routes is then used for the purpose
Final evaluated and then implemented values of weights are shown in Table 3. 


\section{Table 4}

Elasticity's Analysis

\begin{tabular}{|l|ccc|c|}
\hline \multirow{2}{*}{ Scenario name } & \multicolumn{3}{|c|}{ Weights } & \multirow{2}{*}{ Changed routes ratio [\%] } \\
\cline { 2 - 4 } & $\mathbf{A}$ & $\boldsymbol{\beta}$ & $\boldsymbol{\Gamma}$ & \\
\hline -)Standard & 0,7 & 0,02 & $-0,25$ & 52 \\
-)Disregard distance & 0,5 & 0,02 & $-0,45$ & 74 \\
-)Disregard bicycle facilities & 0,8 & 0,02 & $-0,15$ & 39 \\
-) Regard slope and distance & 0,7 & 0,07 & $-0,2$ & 47 \\
\hline
\end{tabular}

The Standard scenario is the commonly one used throughout the whole paper. As expected the highest ratio was observable at the 'disregard distance' - scenario with around three-fourths of all routes changed. The lowest change with 39 percent was in the 'disregard bicycle facilities' - scenario observable.

\subsection{Differences in Shortest Path Distance vs. Attributes Based Routing}

Considering the distance-differences, between purely distance- and attributes based path-finding, a cumulative distribution function was applied, similar as in Aultman et al. (1997), depicted in Fig. 5.

For analysing the results, the distance differences were classified into 50 meterssegments, starting from 0 and ending at $800 \mathrm{~m}$, since, the biggest absolute distancedifference based on the Standard scenario was observed with around $860 \mathrm{~m}$ according to the longest total distance-based path length of around $3 \mathrm{~km}$.

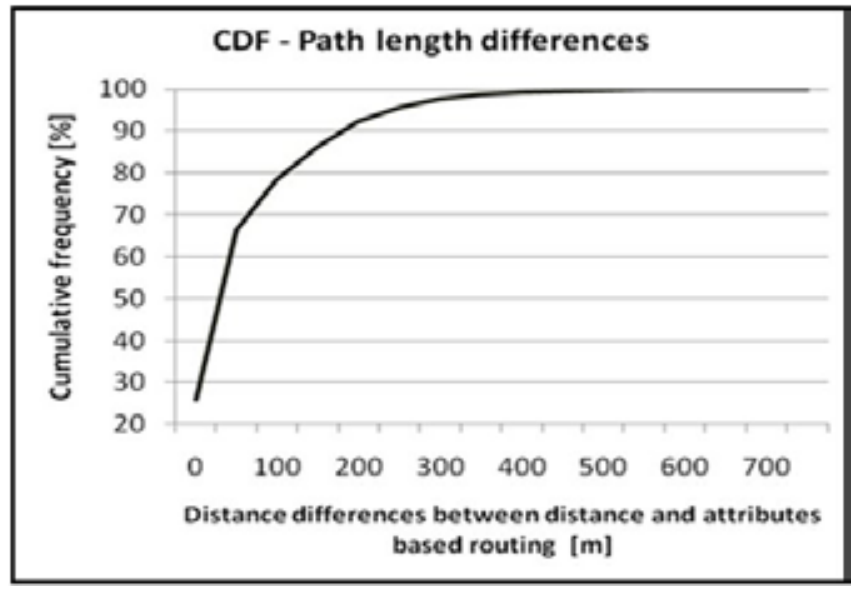

Fig. 5.

Cumulative Distribution Function Applied to Standard-Scenario 
In Fig. 5 it can be clearly seen that around $25 \%$ of all cumulative routes do not show any distance differences between the shortest path distance and attributes based solution. Exemplary paths containing only two nodes show with a very high possibility no alternative routing results than purely distance based. $92 \%$ of all attributes based routes do not show further route extensions of more than $200 \mathrm{~m}$. With the used weights of the Standard scenario, it can be seen that the trade-off between shortest distance and safety respectively comfort can be optimized, fulfilling both criteria (distance and safety/ comfort).

Still the question remains how many extradriven meters a commuter-bicyclist accepts if the security and the comfort should be increased. The answer depends for sure on individual habits of the bicyclists. For instance some might rather take higher slope into account than longer distances. Hereby different weights allow modelling different bicyclists' characteristics.

\section{Bicycle Route Choice Framework}

As described in the introduction besides the modeling results, the project's framework can be implied as a best-practice opportunity to be applied for further bicycle-route-choice investigations. Therefore the general methodology for finding the best-fitting weights for the applied cost function is described in Fig. 6.

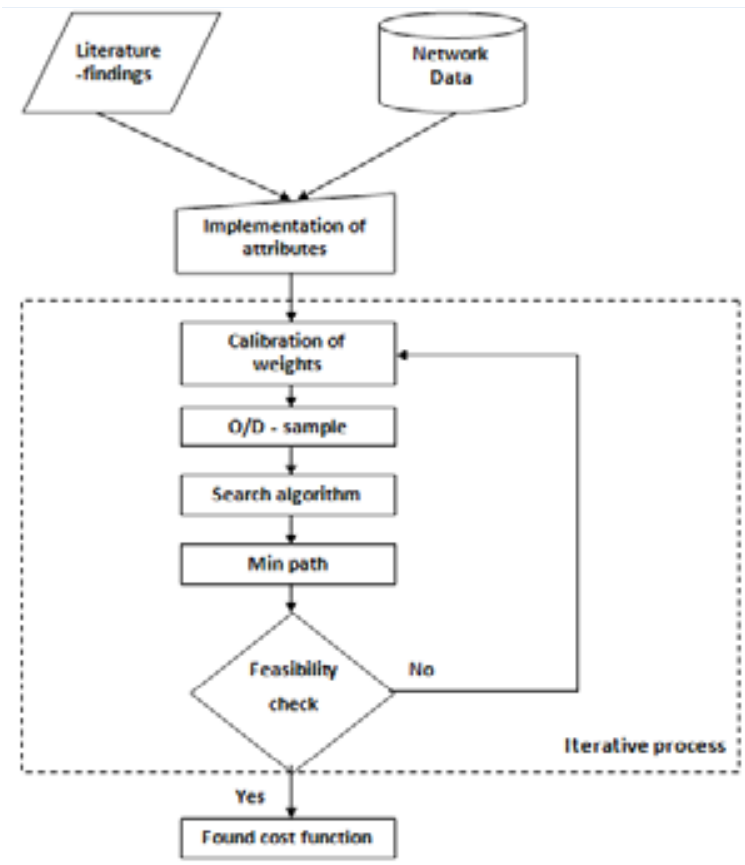

Fig. 6.

Bicycle Route Choice Framework 
The feasibility check itself represents a group discussion of experts. If the group decides for the computed results, an onroad test is started with the given Origin and Destination as in the program specified. If the results of the test-drive matches the algorithm based results than the feasibility check is passed and the found cost function can be defined with the applied weighting factors. Depending on the time there will be checked different Origin/Destinationpairs and evaluated according to the iterative process. In its simple form the group decides for one specific pair of $\mathrm{O} / \mathrm{D}$-pair which has a lot different distinctive features, like different slopes and bicycle lane availability, to compare the algorithms results in terms of pure distance based with the results of attributes based costs.

\section{Conclusions}

This paper is presenting a new model for bicycle route choice. An intense literature survey was carried out, revealing the state-of-the-art in the field. After this study, attributes were chosen and the cost function was carefully developed. The resulting model is capable to predict the most probable path a regular commuter would take between two points located in the defined area. Visualization of the results is properly represented in a network built up with reliable data. The user of the program is able to choose any OD pair and find out the shortest path, the most suitable route according to the cost function, and the elevation profile for each one.

The main advantage of this model is its scalability. It is possible to edit the dataset in order to make it more accurate, enlarge the area or add more attributes. For instance, this model could be adapted in the near future by the Norrköping municipality when planning new investments in infrastructure.

The developed function, able to predict bicyclists routes, can be used afterwards for inexpensive and fast evaluation of possible changes within infrastructure or within traveler information services, for bicycle path routes. Resulting algorithm-framework can be useful for all bikers travelling within the area. Furthermore, the dataset can be enlarged by adding all kinds of information, in order to make it more accurate and reliable. Besides, this network could be used for other modes of transport or any other purpose, avoiding repeating the same data collection process made by the team of this project.

Cycling is a growing mode of transport thanks to its sustainability, safety and healthiness. It is worth to pay more attention to this field and make it more attractive for everyone. This paper improves quality of service for this mode of transport, gives detailed planning information to its users and is able to predict the route choice of the average bicyclist in Norrköping.

\section{References}

AnachB. 2006. Available from Internet: <http://www. anachb.at $/>$.

Aultman-Hall, L.; Hall, F.L.; Baetz, B.B. 1997. Analysis of bicycle commuter routes using geographic information systems: implications for bicycle planning, Transportation Research Record: Journal of the Transportation Research Board. DOI: http://dx.doi.org/10.3141/1578-13, 1578: 102-110.

Broach, J.; Gliebe, J. 2007. Bicycle route choice model developed using revealed preference GPS data, Portland State University, 3-4. 
Cycling Route Planner. 2007. Available from Internet: <http://www.cyclevancouver.ubc.ca/cv.aspx >.

Ehrgotta, M.; Wang, J.Y.T.; Raith, A.; van Houtte, C. 2012. A bi-objective cyclist route choice model, Transportation Research Part A: Policy and Practice. DOI: http://dx.doi.org/10.1016/j.tra.2011.11.015, 46(4): 652-663.

Goldsmith, S. 1992. Reasons Why Bicycling and Walking Are Not Being Used More Extensively As Travel Modes. Publication No. FHWA-PD-92-041, Federal Highway Administration National Bicycle and Walking Study: Case Study\#1 (1992).

Hills, Gradients \& Slopes. In: Bicycle network - Bike future. 2012. Available from Internet: <http://www. bicyclenetwork.com.au/general/bike-futures/91988>.

Hunt, J.D.; Abraham, J.E. 2007. Influences on bicycle use, Transportation.DOI: http://dx.doi.org/10.1007/s11116006-9109-1 , 34(4): 453-470.

Menghini, G.; Carrasco, N.; Schüssler, N.; Axhausen, K.W. 2010. Route choice of cyclists in Zürich, Transportation Research Part A: Policy and Practice. DOI: http://dx.doi.org/10.1016/j.tra.2010.07.008, 44(9): 754-765.
OPT for Health. 2010. Available from Internet: <http:// opt.berkeley.edu/>.

Roberts, K.S. 2012. Measuring Hillnes of Routes. In: Bike Hudson Valley. Available from Internet: <http:// www.roberts-1.com/bikehudson/r/m/hilliness/index. htm $>$.

San Francisco Bicycle trip planner. 2009. Available from Internet: <http://amarpai.com/bikemap/>.

Sener, I.N.; Eluru, N.; Bhat, C.R. 2008. An analysis of bicycle route choice preferences using a web-based survey to examine bicycle facilities. Working Paper, Department of Civil Engineering, University of Texas, Austin.

Stinson, M.; Bhat. C. 2003. An Analysis of Commuter Bicyclist Route Choice Using a Stated Preference Survey, The University of Texas at Austin, Department of Civil Engineering, 6-8.

Transport direct. 2003. Available from Internet: http:// www.transportdirect.info/web2/journeyplanning/ findcycleinput.asp $\mathrm{x}>$.

Westerdijk, P.K. 1990. Pedestrians and Pedal Cyclist Route Choice Criteria, DRIVE Project V1031, Deliverable No. 8B, ITS Working Paper 302. 